\title{
Papel do fator de crescimento vascular endotelial na angiogênese e na retinopatia diabética
}

\author{
The role of vascular endothelial growth factor in \\ angiogenesis and diabetic retinopathy
}

Fabiana Borba Valiatti', Daisy Crispim ${ }^{1,2}$, Camila Benfica ${ }^{3}$, Bruna Borba Valiatti ${ }^{3}$, Caroline K. Kramer ${ }^{1,2}$, Luís Henrique Canani ${ }^{1,2}$

${ }^{1}$ Programa de Pós-Graduação em Ciências Médicas (Endocrinologia), Universidade Federal do Rio Grande do Sul (UFRGS), Porto Alegre, RS, Brasil ${ }^{2}$ Serviço de Endocrinologia, Hospital de Clínicas de Porto Alegre, Porto Alegre, RS, Brasil ${ }^{3}$ Universidade Federal de Ciências da Saúde de Porto Alegre (UFCSPA), Porto Alegre, RS, Brasil

Correspondência para: Fabiana Borba Valiatti Rua Mostardeiro, 5/310 90430-000 - Porto Alegre, RS, Brasil

drafabianavaliatti@hotmail.com

Recebido em 30/Abr/2010 Aceito em 11/Fev/2011

\section{SUMÁRIO}

A retinopatia diabética (RD) é uma complicação microvascular do diabetes melito, sendo importante causa de cegueira adquirida. Fatores angiogênicos, como o vascular endothelial growth factor (VEGF), estão envolvidos na patogênese da RD. O VEGF-A é uma citocina potente e multifuncional que atua por meio dos receptores VEGFR-1 e VEGFR-2 expressos no endotélio vascular causando aumento da permeabilidade vascular e estímulo à neovascularização em processos fisiológicos e patológicos. O VEGFR-2 é o principal mediador mitogênico, angiogênico e do aumento da permeabilidade vascular. Alguns polimorfismos do VEGF têm sido estudados na suscetibilidade e risco de progressão da RD. Importante associação entre o polimorfismo $634 \mathrm{C} / \mathrm{G}$ e a presença de $\mathrm{RD}$ é relatada principalmente em relação ao alelo C. A homozigose $\mathrm{CC}$ estaria relacionada à $\mathrm{RD}$ proliferativa (RDP) e a níveis sérico e vítreo aumentados de VEGF, sugerindo que a presença do alelo $C$ seja um fator de risco independente para RD. Os conhecimentos sobre o VEGF levaram ao desenvolvimento de agentes antiVEGF com o objetivo de inibir a neovascularização patológica e são uma realidade na prática médica do tratamento da RD. Arq Bras Endocrinol Metab. 2011;55(2):106-13

\section{Descritores}

Diabetes melito; retinopatia diabética; fator de crescimento de endotélio vascular; polimorfismo

\section{SUMMARY}

Diabetic retinopathy (DR), a DM microvascular complication, is the leading cause of blindness. Angiogenic factors such as vascular endothelial growth factor (VEGF) are involved in the pathogenesis of DR. VEGF-A is a potent, multifunctional cytokine that acts through the receptors VEGFR-1 and VEGFR-2 expressed in the vascular endothelium and causing increased vascular permeability and neovascularization stimulation in both physiological and pathological processes. The expression of VEGFR-1 is upregulated by hypoxia and is less responsive to VEGF compared to VEGFR-2 which is the main mediator mitogenic, angiogenic, and increased vascular permeability. VEGF polymorphisms have been studied in DR susceptibility and progression. Significant association between the polymorphism $634 \mathrm{C} / \mathrm{G}$ and the presence of RD is reported mainly in relation to allele $\mathrm{C}$. The homozygous $\mathrm{CC}$ is associated to proliferative $\mathrm{RD}$ and to increased vitreous and serum levels of VEGF suggesting that the presence of the $\mathrm{C}$ allele is an independent risk factor for RD. The knowledgement of VEGF lead to the development of anti-VEGF drugs (pegaptanib, ranibizumab and bevacizumab) aiming to prevent pathological neovascularization. The anti-VEGF therapy is a reality in practice medical treatment of DR. Arq Bras Endocrinol Metab. 2011;55(2):106-13

\section{Keywords}

Diabetes mellitus; diabetic retinopathy; vascular endothelial growth factor; polymorphism 
$\mathrm{E}$ stima-se que $7,6 \%$ da população brasileira seja portadora de diabetes melito (DM) $(1,2)$. As complicações microvasculares são as maiores responsáveis pela morbidade no DM, sendo a retinopatia diabética $(\mathrm{RD})$ a principal causa de cegueira adquirida em adultos (3).

No momento do diagnóstico, cerca de $21 \%$ dos pacientes com DM tipo 2 apresentam alguma evidência de $\mathrm{RD}$ (3). Os principais fatores de risco para o desenvolvimento da RD são tempo de doença (3), hiperglicemia $(4,5)$ e pressão arterial elevada $(5)$. Entretanto, alguns indivíduos não desenvolvem $\mathrm{RD}$ mesmo após exposição prolongada à hiperglicemia e à hipertensão arterial $(6,7)$, sugerindo que os mecanismos dessa doença ainda não estão completamente esclarecidos.

Dentre os fatores potencialmente envolvidos na patogênese da $\mathrm{RD}$, destacam-se fatores angiogênicos como o vascular endothelial growth factor (VEGF). Dessa forma, nosso objetivo é revisar o papel da angiogênese e do VEGF em relação à RD.

\section{RETINOPATIA DIABÉTICA}

A RD é uma desordem da vascularização retiniana caracterizada por anormalidades microvasculares (microaneurismas e hemorragias intrarretinianas), progredindo para alteração da permeabilidade vascular, má perfusão tecidual, edema e isquemia retiniana e anormalidades microvasculares intrarretinianas (IRMAs) (8). A fase avançada, associada ao aumento do risco de cegueira, é chamada $\mathrm{RD}$ proliferativa $(\mathrm{RDP})$ e determinada pela presença de neovascularização da retina ou do disco óptico (8) (Tabela 1).

Tabela 1. Classificação Internacional de Severidade da Retinopatia Diabética

\begin{tabular}{|c|c|}
\hline Severidade da doença & Achados oftalmoscópicos sob midríase \\
\hline Ausência de retinopatia aparente & Ausência de anormalidades \\
\hline RD leve não proliferativa & Apenas microaneurismas \\
\hline RD moderada não proliferativa & $\begin{array}{l}\text { Mais que apenas microaneurismas, mas } \\
\text { menos que RD severa não proliferativa }\end{array}$ \\
\hline RD severa não proliferativa & $\begin{array}{l}\text { Sem sinais de RD proliferativa, com } \\
\text { qualquer dos achados abaixo: } \\
\text { - Mais de } 20 \text { hemorragias intrarretinianas } \\
\text { em cada um dos quatro quadrantes } \\
\text { - Veias em rosário em pelo menos dois } \\
\text { quadrantes } \\
\text { - Anormalidades microvasculares } \\
\text { intrarretinianas em pelos menos um } \\
\text { quadrante }\end{array}$ \\
\hline RD proliferativa & $\begin{array}{l}\text { Qualquer dos achados abaixo: } \\
\text { - Neovascularização } \\
\text { - Hemorragia vitrea ou pré-retiniana }\end{array}$ \\
\hline
\end{tabular}

RD: retinopatia diabética.

Adaptado de Wilkinson CP, et al. (9).
Os neovasos presentes na RDP são frágeis e podem sangrar para cavidade vítrea, o que altera a estrutura vitreorretiniana acarretando fibrose e tração sobre a retina. As complicações, a partir desse momento, incluem descolamento tracional e rupturas na retina. A evolução dessa doença pode resultar em perda importante e irreversível da visão $(10,11)$.

Em 1948, Michaelson (12) propôs a existência de um fator difusível desconhecido produzido pela retina isquêmica e responsável pela angiogênese que causaria neovascularização da retina e da íris, como ocorre na RDP e outras doenças vasculares da retina (12). Assim, o entendimento da angiogênese e fatores envolvidos ganham importância no conhecimento da patogênese da RD.

\section{ANGIOGÊNESE}

A angiogênese é um processo fundamental e complexo no qual ocorre formação de novos vasos sanguíneos a partir de vasos preexistentes (13-18), sendo essencial em condições fisiológicas como ovulação, desenvolvimento do corpo lúteo, embriogênese, crescimento tecidual, desenvolvimento mamário na lactação, resposta imune, inflamação e cicatrização. No adulto saudável, o turnover vascular é extremamente baixo e a angiogênese raramente ocorre $(13,14,19)$.

A etiologia e a patogênese de algumas doenças são determinadas pela resposta angiogênica persistente devido a um aumento dos mediadores angiogênicos ou deficiência dos inibidores da angiogênese, como, por exemplo, neoplasias, metástases, psoríase e artrite reumatoide, entre outras $(13,14,16)$. Nessas situações, a angiogênese é altamente regulada com períodos de ativação e inibição $(13,16)$. Doenças oculares como a $\mathrm{RD}$, o glaucoma neovascular, as oclusões vasculares, a retinopatia da prematuridade e a degeneração macular relacionada à idade também apresentam alteração da angiogênese $(13,14,16,18,20)$.

Os vasos alvos dos fatores angiogênicos são os capilares venosos e as vênulas terminais, os quais apresentam pequeno calibre com células endoteliais sobre uma lâmina basal cobertas por uma camada descontínua de pericitos e células musculares lisas (21). Um dos processos iniciais da resposta angiogênica é a quebra das ligações entre as células dessas camadas (16). As células endoteliais ativadas geram enzimas proteolíticas que permitem a degradação da matriz extracelular e migração dessas células através da membrana basal, a partir do vaso da qual se originam, expressando moléculas de adesão na superfície celular (22). 


\section{FATORES ENVOLVIDOS NA ANGIOGÊNESE}

Vários peptídeos com papel de fator de crescimento relacionados à angiogênese já foram purificados, entre eles o fator ácido de crescimento de fibroblastos (aFGF), o fator básico de crescimento de fibroblastos (bFGF), a angiogenina, o fator endotelial II derivado das plaquetas (PD-ECGF), o fator induzido por hipóxia (HIF-1), o fator de transformação do crescimento $\alpha$ e $\beta$ (TGF- $\alpha$ e TGF- $\beta$ ), o fator de crescimento derivado de plaqueta $(P D G F)$, o fator de necrose tumoral $\alpha$ (TNF- $\alpha)$, o fator de crescimento epidérmico $(E G F)$, a interleucina 1 ( $I L$ l), a interleucina 2 ( $I L-2)$, o fator de cicatrização/fator de crescimento do hepatócito (SF/HGF) e o fator de permeabilidade vascular (VPF), atualmente chamado de fator de crescimento do endotélio vascular (VEGF) (13,16,23-25).

Dentre esses fatores, o VEGF é um dos mais investigados atualmente. O VEGF pertence a um grupo de glicoproteínas diméricas que inclui o fator de crescimento placentário (PIGF), VEGF-A, VEGF-B, VEGF-C, VEGF-D, VEGF-E e VEGF-F (18,26). O VEGF-A é uma citocina potente e multifuncional que exerce seu efeito no endotélio $(17,27)$, sendo o mais bem estudado e compreendido. Nesta revisão, sempre que for mencionado o VEGF, nos referiremos ao VEGF-A (26).

$\mathrm{O}$ gene humano do VEGF- $A$ está organizado em oito éxons, separados por sete íntrons e localizado no cromossomo $6 \mathrm{p} 21.3$ (28). O splicing alternativo resulta em quatro isoformas principais, contendo 121, 165, 189 e 206 aminoácidos, respectivamente, VEGF121, VEGF165, VEGF189, VEGF206 (17,23,24,29). Embora com menor frequência, outras variantes também têm sido relatadas, incluindo VEGFl45, VEGF183, VEGF162 e VEGF165b. Paradoxalmente, esta última variante teria um efeito inibitório na mitogênese induzida pelo VEGF (30).

O VEGF165, a principal isoforma VEGF-A $(17,18)$, é uma glicoproteína homodimérica de $45 \mathrm{kDa}$ que se liga à heparina. Após ser secretada, uma fração significativa permanece vinculada à superfície celular e matriz extracelular (18).

O VEGF atua direta e seletivamente através dos receptores VEGFR-1 e VEGFR-2, expressados predominantemente, e talvez exclusivamente, no endotélio vascular (17). A ligação do VEGF a esses receptores causa influxo de cálcio citoplasmático, aumentando sua concentração em até quatro vezes, mudança da forma, divisão e migração celular (17,18,31-33). Esse aumento da permeabilidade das vênulas às macromoléculas permite que proteínas plasmáticas extravasem para o espaço extravascular, levando a coagulação do fibrinogênio e deposição de gel de fibrina que serve como matriz provisória para o crescimento de novos vasos sanguíneos $(15,17,18,23)$. O aumento da permeabilidade microvascular parece, invariavelmente, preceder e/ou acompanhar a angiogênese numa variedade de processos fisiológicos e patológicos $(17,24)$. Isso faz com que o VEGF seja considerado um importante mediador de angiogênese.

In vitro o VEGF é capaz para promover o crescimento de células endoteliais vasculares a partir de artérias, veias e vasos linfáticos e impede a apoptose endotelial induzida por privação de nutrientes. Além disso, tanto in vitro quanto in vivo o VEGF também é um fator de sobrevivência para o endotélio (27). Em modelo experimental, a inibição do VEGF resulta em grandes alterações de apoptose na vasculatura neonatal, mas não em adultos (34). Parece haver maior dependência do VEGF nas células endoteliais recém-formadas, mas não nos vasos já estabelecidos dentro dos tumores (34). A cobertura dos vasos por pericitos foi proposta como um dos principais eventos para a perda da suscetibilidade ao VEGF (21).

\section{RECEPTORES DO VEGF}

Três receptores do VEGF são descritos: VEFGR-1, VEGFR-2 e VEGFR-3. As proteínas transmembrana VEGFR-1 (Flt-1) e VEGFR-2 (Flk-1/KDR) são receptores VEGF de alta afinidade com domínio de tirosina quinase $(17,18,31)$. O VEGFR-3 (Flt-4) é membro da mesma família de receptores tirosina quinase, mas tem afinidade pelo VEGF-C e pelo VEGF-D, não sendo um receptor de VEGF-A (18).

A expressão do VEGFR-1 (Flt-1: fms-like da tirosina quinase) é acentuada por hipóxia por meio de mecanismo dependente do fator induzido pela hipóxia (HIF-1). O VEGFR-1 tem afinidade pelo VEGF-A, mas também PIGF e VEGF-B, no entanto, o VEGFR-1 apresenta fraca resposta ao VEGF (18). Park e cols. (35) inicialmente propuseram que o VEGFR-1 não seria essencialmente um receptor de transmissão de um sinal mitogênico, mas um receptor modulador capaz de regular de forma negativa a atividade do VEGF no endotélio vascular, sequestrando-o e tornando esse fator menos disponível para o VEGFR-2. Assim, a potencialização da ação do VEGF por PIGF poderia ser explicada, pelo menos em parte, pelo deslocamento do VEGF 
do VEGFR-1 ligado (35). Ativação do VEGFR-1 por PlGF resultaria em transfosforilação de VEGFR-2, assim amplificaria angiogênese causada pelo VEGF através VEGFR-2 (18).

Podemos considerar que o VEGFR-1 tenha dupla função na angiogênese, uma positiva e outra negativa, em circunstâncias diferentes (36).

O VEGF se liga ao VEGFR-2 (KDR, humanos; Flk-1, camundongo), embora com menor afinidade do que ao VEGFR-1, tendo papel no desenvolvimento da angiogênese e hematopoiese (36). Existe consenso de que o VEGFR-2 é o principal mediador mitogênico, angiogênico e do aumento da permeabilidade vascular devido ao VEGF $(18,23,33,36)$.

\section{REGULAÇÃO DA EXPRESSÃO GÊNICA DO VEGF}

A expressão do RNA mensageiro (RNAm) VEGF é induzida pela exposição à baixa tensão de oxigênio em uma variedade de circunstâncias patológicas $(17,18)$. O VEGF tem sua expressão aumentada quando medido por meio do RNAm ou da proteína em vários tumores malignos em animais e humanos (17). A hipóxia tecidual tem sido associada com à angiogênese e há evidências de que a concentração de oxigênio local regula a expressão do VEGF (17), podendo ela ser o principal indutor da transcrição do gene do $\operatorname{VEGF}(18)$.

\section{VEGF E DOENÇA OCULAR NO DIABETES MELITO}

Todos os conceitos expostos anteriormente se aplicam amplamente à $\mathrm{RD}$ e têm sido motivo de inúmeros estudos que objetivam encontrar marcadores que possam predizer a evolução dessa doença e assim ser alvo de tratamento.

Em 1948, Michaelson (12) postulou a existência de um fator angiogênico difusível, liberado pela retina isquêmica. Por ser induzido por hipóxia, VEGF tornou-se um candidato atraente como mediador de neovascularização intraocular patológica.

O VEGF tem sido investigado como um fator de crescimento com níveis aumentados no humor aquoso e vítreo de pacientes com $\mathrm{RD}$, principalmente naqueles com RDP (37-39). Pacientes com DM e RDP apresentam níveis de VEGF no humor aquoso e vítreo maiores que no plasma, o que possivelmente está relacionado à atividade da $\mathrm{RD}$. O mesmo se observa quanto ao edema macular em pacientes diabéticos (37-39).
Vários estudos acerca da presença de edema macular e sua relação com os níveis de VEGF também têm sido realizados. Patel e cols. (40) observaram correlação positiva entre o maior nível de VEGF e a ocorrência de edema macular quando a mácula foi analisada com tomografia de coerência óptica (Optical Coherence Tomography - OCT). Funatsu e cols. (41), em estudo prospectivo de pacientes com $\mathrm{RD}$ não proliferativa submetidos à facoemulsificação do cristalino, verificaram que níveis elevados de VEGF no humor aquoso medidos mediante coleta de amostra no início do procedimento estavam associados com piora do edema macular no pós-operatório. Assim, o nível do VEGF poderia ser tomado como fator de risco para exacerbação do edema macular em pacientes com DM submetidos à cirurgia de catarata.

As concentrações intraoculares de VEGF diminuem em pacientes com RDP submetidos à fotocoagulação com laser da retina, provavelmente pela diminuição do estímulo isquêmico e, consequentemente, diminuição do estímulo à angiogênese (42).

\section{POLIMORFISMOS DO VEGF NA RETINOPATIA DIABÉTICA}

Alguns polimorfismos do VEGF têm sido estudados para avaliar sua relação com a suscetibilidade e risco de progressão da RD (Tabela 2). Errera e cols. (43) não observaram associação entre do polimorfismo $-634 \mathrm{G} / \mathrm{C}$ (rs2010963) do VEGF e a presença de RD ou de DM tipo 2. Entretanto, a homozigose CC no polimorfismo $-634 \mathrm{G} / \mathrm{C}$ foi mais frequente nos pacientes com RDP quando comparado ao grupo controle (pacientes diabéticos tipo 2 sem RDP), sugerindo que a presença de homozigose do alelo -634C do VEGF seja um fator de risco independente para RDP em pacientes com DM tipo 2 (43).

Diante das evidências de que o VEGF tenha importante papel na patogênese da $\mathrm{RD}$, um estudo avaliou quatro polimorfismos do VEGF já previamente descritos $-7 \mathrm{C} / \mathrm{T}(\mathrm{rs} 25648)$ e $-634 \mathrm{C} / \mathrm{G}$ na região 5' untranslated region (UTR) e $-1498 \mathrm{~T} / \mathrm{C}$ e $-1190 \mathrm{G} / \mathrm{A}$ na região promotora do $V E G F$ e sua possível relação com $\mathrm{RD}$ em população indiana com DM tipo 2 . A frequência dos polimorfismos -7C/T, - 1498T/C e -634C/G teve diferenças significativas entre os pacientes com $\mathrm{RDP}$ e sem sinais de $\mathrm{RD}$ ou $\mathrm{RD}$ leve. Isso sugere que esses polimorfismos podem estar associados ao maior risco de $\mathrm{RD}(8)$. 
Tabela 2. Estudos de polimorfismos do VEGF

\begin{tabular}{|c|c|c|c|c|}
\hline População & Amostra & Polimorfismo & Observação & Ref. \\
\hline Coreana & 398 com DM2, 526 sem DM & $\begin{array}{c}+936 \mathrm{C} / \mathrm{T} \\
(\mathrm{rs} 3025039)\end{array}$ & $\begin{array}{c}\text { Alelo T mais frequente em DM2 com RD }(P=0,042) \\
\text { Alelo T associado com níveis mais altos VEGF plasmático }(P<0,05)\end{array}$ & (44) \\
\hline \multirow[t]{5}{*}{ Finlandesa } & $\begin{array}{l}131 \text { DM } 1 \text { e } 2 \text { com RD, } 98 \text { DM } \\
1 \text { e } 2 \text { sem RD, } 526 \text { sem DM }\end{array}$ & $\begin{array}{l}-2578 \mathrm{C} / \mathrm{A} \\
(\mathrm{rs} 699947)\end{array}$ & Não houve associação com RD & (45) \\
\hline & & $\begin{array}{l}-634 C / G \\
(\text { rs2010963) }\end{array}$ & Não houve associação com RD & (45) \\
\hline & & rs2146232 & Não houve associação com RD & (45) \\
\hline & & rs3025033 & Não houve associação com RD & (45) \\
\hline & & $\begin{array}{l}+936 \mathrm{C} / \mathrm{T} \\
(\mathrm{rs} 3025039)\end{array}$ & Não houve associação com RD & (45) \\
\hline \multirow[t]{2}{*}{ Japonesa } & 175 DM1 & rs833070 & $\begin{array}{c}\text { Genótipo TT associado à progressão precoce para RDNP severa RC: } 1,67 \text { (IC } \\
\text { 95\%:1.01-2,54) }(\mathrm{P}=0,047)\end{array}$ & (46) \\
\hline & & rs2146323 & $\begin{array}{l}\text { Genótipo AA associado à progressão precoce para RDNP severa RC: } 1,68 \text { (IC } \\
\text { 95\%: } 1.02-2,57)(P=0,043)\end{array}$ & (46) \\
\hline \multirow[t]{2}{*}{ Japonesa } & 469 DM2 & $\begin{array}{l}-634 C / G \\
(\text { rs2010963) }\end{array}$ & Não houve associação com RD & $(47)$ \\
\hline & & $\begin{array}{l}-2578 C / A \\
(\text { rs699947) }\end{array}$ & $\begin{array}{l}\text { Maior frequência do alelo A no grupo RDP quando comparado ao controle ( } \mathrm{P}=0,036) \\
\text { Genótipo AA RC: } 7,7 \text { (IC 95\%: 1,8-30,9) }\end{array}$ & $(47)$ \\
\hline \multirow[t]{3}{*}{ Britânica } & $\begin{array}{c}\text { DM 1 e 2: } 45 \text { com RDP, } 61 \text { sem } \\
\text { RD }\end{array}$ & $-160 \mathrm{C} / \mathrm{T}$ & $\begin{array}{c}\text { Associação significativa e independente com RDP } \\
\text { CC teve RC 10,5 (2,3-47,7) para RDP } \\
\text { P }=0,0003\end{array}$ & $(48)$ \\
\hline & & $\begin{array}{c}-152 \mathrm{~A} / \mathrm{G} \\
(\mathrm{rs} 13207351)\end{array}$ & $\begin{array}{l}\text { Associação significativa e independente com RDP } \\
\text { AA teve RC } 3,5(1,5-7,7) \text { para RDP } \\
\text { P=0,0022 }\end{array}$ & $(48)$ \\
\hline & & $-116 A$ & $\begin{array}{c}\text { Associação significativa e independente com RDP } \\
\text { AA teve RC } 7,9(3,1-19,9) \text { para RDP } \\
\qquad P=3,23 \times 10^{-6}\end{array}$ & $(48)$ \\
\hline Eslovênica & $\begin{array}{l}206 \text { DM } 2 \text { com RDP e } 143 \text { DM2 } \\
\quad>10 \text { anos sem RD }\end{array}$ & $\begin{array}{l}-634 C / G \\
(\text { rs } 2010963)\end{array}$ & $\begin{array}{l}\text { RDP não esteve associada com o polimorfismo } \\
\text { Níveis sérico e vítreo de VEGF foram maiores no genótipo } \mathrm{CC} P<0,001\end{array}$ & (49) \\
\hline \multirow[t]{2}{*}{ Indiana } & DM2 com e sem RD & $\begin{array}{l}-634 C / G \\
\text { (rs2010963) }\end{array}$ & $\begin{array}{l}\text { Não houve associação com presença ou severidade de RD, no entanto esse } \\
\text { polimorfismo foi associado com aumento do risco de RD em pacientes com } \\
\text { microalbuminúria (RC: } 8,9, \text { IC } 95 \%: 1,4-58,3 \text { ) }\end{array}$ & (50) \\
\hline & & $\begin{array}{l}+936 \mathrm{C} / \mathrm{T} \\
(\mathrm{rs} 3025039)\end{array}$ & Não houve associação com RD & $(50)$ \\
\hline \multirow[t]{2}{*}{ Polonesa } & $\begin{array}{l}\text { DM2: } 82 \text { RDP, } 72 \text { RDNP, } 61 \\
\text { sem RD (controle) }\end{array}$ & $\begin{array}{l}-460 \mathrm{C} / \mathrm{T} \\
(\mathrm{rs} 833061)\end{array}$ & Não houve associação com RD & $(51)$ \\
\hline & & $\begin{array}{l}-634 C / G \\
\text { (rs2010963) }\end{array}$ & $\begin{array}{l}\text { Alelo C foi mais frequente em RDNP quando comparado ao controle }(\mathrm{RC}=1,69 \text {, } \\
\qquad \text { IC } 95 \% 1,03-2,79)\end{array}$ & $(51)$ \\
\hline Brasileira & DM2: 167 RDP, 334 sem RDP & $\begin{array}{l}-634 C / G \\
(\text { rs2010963) }\end{array}$ & $\begin{array}{c}\text { Alelo C em homozigose foi mais frequente em RDP quando comparada ao } \\
\text { controle (RC: } 1,9, \text { IC } 95 \%: 1,01-3,79)(P=0,04)\end{array}$ & (43) \\
\hline \multirow[t]{3}{*}{ Indiana } & $\begin{array}{l}\text { DM2: } 120 \text { com RDP e } 90>15 \\
\text { anos sem RD ou RD leve }\end{array}$ & $\begin{array}{l}-7 \mathrm{C} / \mathrm{T} \\
(\mathrm{rs} 25648)\end{array}$ & Genótipo CT associado à RD (RC: 4,17, IC 95\%: 1,90-9,18) (P=0,0001) & (8) \\
\hline & & $\begin{array}{l}-634 C / G \\
\text { (rs2010963) }\end{array}$ & Genótipo CG associado à RD (RC: 4,37, IC 95\%: 2,44-7,84) (P=0,0001) & (8) \\
\hline & & $-1498 \mathrm{~T} / \mathrm{C}$ & Genótipo TC associado à RD (RC: 2,33, IC 95\%: 1,24-4,36) (P=0,0008) & (8) \\
\hline \multirow[t]{3}{*}{ Japonesa } & $\begin{array}{l}\text { DM2: } 203 \text { sem RD, } 93 \text { RDNP, } \\
82 \text { RDP }\end{array}$ & $\begin{array}{l}-2578 \mathrm{C} / \mathrm{A} \\
(\mathrm{rs} 699947)\end{array}$ & Não houve associação com RD & $(52)$ \\
\hline & & $\begin{array}{l}-1154 G / A \\
(\text { rs } 1570360)\end{array}$ & Não houve associação com RD & $(52)$ \\
\hline & & $\begin{array}{l}-634 C / G \\
(\text { rs2010963) }\end{array}$ & $\begin{array}{l}\text { Alelo } C \text { considerado fator de risco para EMD, independente da presença de RD } \\
\qquad(P=0,047) \text {, e para } R D(P=0,02)\end{array}$ & $(52)$ \\
\hline Britânica & $\begin{array}{l}\text { DM1 e 2: } 69 \text { RDP, } 198 \text { outros } \\
\text { graus de RD }\end{array}$ & $\begin{array}{l}-460 \mathrm{C} / \mathrm{T} \\
(\mathrm{rs} 833061)\end{array}$ & Alelo C esteve associado a RDP (RC: 2,5, IC 95\%:1,20-5,23) & $(53)$ \\
\hline Japonesa & $\begin{array}{c}\text { DM2: } 150 \text { com RD, } 118 \text { sem } \\
\text { RD }\end{array}$ & $\begin{array}{l}-634 C / G \\
(\text { rs2010963) }\end{array}$ & $\begin{array}{l}\text { Alelo C foi mais frequente em RD quando comparado aos sem } R D(P=0,0037) \\
\text { A RC entre o genótipo CC e o GG foi 3,2 (IC 95\%: } 1,45-7,05)(P=0,0046) \\
\text { Alelo C foi mais frequente em RDNP }(P=0,0026) \text { do que em } \operatorname{RDP}(P=0,081)\end{array}$ & $(54)$ \\
\hline
\end{tabular}

DM: diabetes melito; DM1: diabetes melito tipo 1; DM2: diabetes melito tipo 2; RDP: retinopatia diabética proliferativa; VEGF: vascular endothelial growth factor; RD: retinopatia diabética; RDNP: retinopatia diabética não proliferativa; EMD: edema macular diabético; RC: razão de chance; IC: intervalo de confiança. 
Awata e cols. (54) identificaram seis polimorfismos do VEGF em japoneses com DM tipo 2, sendo que os $-1877 \mathrm{G} / \mathrm{A},-1498 \mathrm{~T} / \mathrm{C},-1190 \mathrm{G} / \mathrm{A}$ e $-1154 \mathrm{G} / \mathrm{A}$ (rs1570360) estão localizados na região promotora e $-634 \mathrm{C} / \mathrm{G}$ e $-7 \mathrm{C} / \mathrm{T}$ na 5' UTR. O polimorfismo - 1877G/A demonstrou-se raro e sua avaliação não foi continuada. A distribuição do polimorfismo $-634 \mathrm{C} / \mathrm{G}$ foi relacionada à $\mathrm{RD}$, sugerindo a presença do alelo $\mathrm{C}$ que seja um fator de risco independente pra $\mathrm{RD}$. O genótipo CC teve odds ratio de 3,2 quando comparado ao genótipo GG. Inicialmente, Awata e cols. (54) acreditaram que alguns polimorfismos do VEGF poderiam estar associados aos estágios mais avançados de $\mathrm{RD}$ como a forma proliferativa, já que o VEGF é um forte fator angiogênico. No entanto, o polimorfismo $634 \mathrm{C} / \mathrm{G}$ não esteve especificamente associado com RDP, pelo contrário, a associação foi mais evidente na $\mathrm{RD}$ não proliferativa.

Petrovic e cols. (49) não observaram associação entre RDP e o polimorfismo $-634 \mathrm{C} / \mathrm{G}$ do $V E G F$ em pacientes com DM tipo 2. No entanto, a média dos níveis séricos e vítreos de VEGF foi significativamente maior nos pacientes com RDP quando comparados ao grupo controle (diabéticos sem RD). Esses níveis foram significativamente maiores em diabéticos com o genótipo CC, quando comparados aos outros genótipos (CG e GG).

Estudos populacionais mostram que não há diferença importante entre as etnias na distribuição dos alelos C e G do polimorfismo - $634 \mathrm{C} / \mathrm{G}$, sendo o alelo $\mathrm{G}$ mais frequente em todas as populações estudadas (55). Ao avaliar três polimorfismos na região promotora do gene VEGF, -2578C/A (rs699947), -1154 G/A e -634C/ $\mathrm{G}$, pesquisadores de Campinas/SP observaram que, embora as variantes $-2578 \mathrm{~A} \mathrm{e}-1154 \mathrm{~A}$ sejam mais comuns em brancos que em negros, não houve diferenças significativas interétnicas em relação ao polimorfismo $-634 \mathrm{C} / \mathrm{G}(56)$.

\section{INTERVENÇÃO NO VEGF COMO TRATAMENTO DA RETINOPATIA DIABÉTICA}

Os primeiros estudos sobre VEGF e RD são da década de 1980 e ainda é de grande interesse médico devido à mudança do caráter meramente investigativo para propósitos terapêuticos focados nesse fator.

O conhecimento dos conceitos acima abriu um importante campo de investigação, tendo como alvo o VEGF na busca de uma terapia que anulasse seus efeitos patológicos. Recentemente, agentes antiVEGF foram desenvolvidos com objetivo de inibir a neovascularização patológica. Esse interesse, obviamente, envolveu a DM e suas complicações como a RD.

O pegaptanibe $\left(\right.$ Macugen $\left.^{\circledR}\right)$, o ranibizumabe $\left(\mathrm{Lu}^{-}\right.$ centis $^{\circledR}$ ) e o bevacizumabe (Avastin ${ }^{\circledR}$ ) são as medicações antiVEGF disponíveis atualmente. O ranibizumabe e o bevacizumabe bloqueiam todas as isoformas circulantes de VEGF, inibindo a neovascularização em condições fisiológicas e patológicas. O pegaptanibe age seletivamente no VEGF165. No tratamento da RD, essas medicações são aplicadas dentro da cavidade vítrea. Em decorrência da curta duração do seu efeito, os antiVEGFs necessitariam de injeções intravítreas repetidas, o que aumentaria o risco de endoftalmite, aumento da pressão intraocular, rasgo de epitélio pigmentar, rasgo e descolamento de retina. Apesar de ainda não ser bem investigado, em estudo recente foi observado aumento da pressão arterial nos pacientes normotensos e persistência da hipertensão em pacientes com HAS submetidos à injeção intravítrea de bevacizumabe (57).

A terapia antiVEGF, apesar de ainda necessitar de estudos clínicos e ter uso off-label na $\mathrm{RD}$, é uma realidade na prática médica do tratamento dessa doença $(58,59)$.

Em conclusão, é fundamental pensarmos na angiogênese ocular, tendo como maior exemplo a $\mathrm{RD}$ como um processo multifatorial. A identificação de fatores, como o VEGF, que tenham papel importante no processo de angiogênese fisiológica e patológica estimula o desenvolvimento de estudos que investiguem ainda mais a fisiopatogenia das doenças vasculares da retina bem como apontem para novas possibilidades terapêuticas.

Agradecimentos: Ao Fundo de Incentivo à Pesquisa e Eventos do Hospital de Clínicas de Porto Alegre (FIPE-HCPA) e ao Conselho Nacional de Desenvolvimento Científico e Tecnológico (CNPq).

Declaração: os autores declaram não haver conflitos de interesse científico neste estudo.

\section{REFERÊNCIAS}

1. Rede Interagencial de Informações para a Saúde - RIPSA. Disponível em: http://tabnet.datasus.gov.br/cgi/idb2008/d10.htm. Acesso em: Mar 20, 2010.

2. Malerbi DA, Franco LJ. Multicenter study of the prevalence of diabetes mellitus and impaired glucose tolerance in the urban Brazilian population aged 30-69 yr. The Brazilian Cooperative Group on the Study of Diabetes Prevalence. Diabetes Care. 1992;15(11):1509-16.

3. Fong DS, Aiello L, GardnerTW, King GL, Blankenship G, Cavallerano JD, et al. Diabetic retinopathy. Diabetes Care. 2003;26 Suppl 1:S99-S102. 
4. UK Prospective Diabetes Study (UKPDS) Group. Intensive blood-glucose control with sulphonylureas or insulin compared with conventional treatment and risk of complications in patients with type 2 diabetes (UKPDS 33). Lancet. 1998;352(9131):837-53.

5. Stratton IM, Kohner EM, Aldington SJ, Turner RC, Holman RR, Manley SE, et al., UKPDS 50: risk factors for incidence and progression of retinopathy in Type II diabetes over 6 years from diagnosis. Diabetologia. 2001;44(2):156-63.

6. Esteves JLA, Roggia MF, Dalpizol M, Scocco C, Kramer CK, Azevedo $\mathrm{MJ}$, et al. Fatores de risco para retinopatia diabética. Arq Bras Endocrinol Metab. 2008;52(3):431-41.

7. Klein R, Klein BE, Moss SE, Davis MD, DeMets DL. The Wisconsin epidemiologic study of diabetic retinopathy. III. Prevalence and risk of diabetic retinopathy when age at diagnosis is 30 or more years. Arch Ophthalmol. 1984;102(4):527-32.

8. Suganthalakshmi B, Anand R, Kim R, Mahalakshmi R, Karthikprakash $\mathrm{S}$, Namperumalsamy $\mathrm{P}$, et al. Association of VEGF and eNOS gene polymorphisms in type 2 diabetic retinopathy. Mol Vis. 2006(12):336-41.

9. Wilkinson $\mathrm{CP}$, Ferris FL, Klein RE, et al. Proposed international clinical diabetic retinopathy and diabetic macular edema disease severity scales. Ophthalmology. 2003;110(9):1677-82.

10. CiullaTA, Amador AG, Zinman B. Diabetic retinopathy and diabetic macular edema: pathophysiology, screening, and novel therapies. Diabetes Care. 2003;26(9):2653-64.

11. Aiello LP, Gardner TW, King GL, Blankenship G, Cavallerano JD, Ferris FL, et al. Diabetic retinopathy. Diabetes Care. 1998;21(1):143-56.

12. Michaelson I.The mode of development of the vascular system of the retina with some observations on its significance for certain retinal disorders. Trans Ophthalmol Soc UK. 1948(68):137-80.

13. Folkman J, Shing Y. Angiogenesis. J Biol Chem. 1992;267(16): 10931-4.

14. Zetter BR. Angiogenesis. State of the art. Chest. 1988;93(3 Suppl):159S-66S.

15. Folkman J. How is blood vessel growth regulated in normal and neoplastic tissue? G.H.A. Clowes memorial Award lecture. Cancer Res. 1986;46(2):467-73.

16. Polverini PJ. The pathophysiology of angiogenesis. Crit Rev Oral Biol Med. 1995;6(3):230-47.

17. Dvorak HF, Brown LF, Detmar M, Dvorak AM. Vascular permeability factor/vascular endothelial growth factor, microvascular hyperpermeability, and angiogenesis. Am J Pathol. 1995;146(5): 1029-39.

18. Ferrara N. Vascular endothelial growth factor: basic science and clinical progress. Endocr Rev. 2004;25(4):581-611.

19. Benjamin LE, Golijanin D, Itin A, Pode D, Keshet E. Selective ablation of immature blood vessels in established human tumors follows vascular endothelial growth factor withdrawal. J Clin Invest. 1999;103(2):159-65.

20. Miller JW. Vascular endothelial growth factor and ocular neovascularization. Am J Pathol. 1997;151(1):13-23.

21. Hellstrom M, Gerhardt $H$, Kalén M, Li X, Eriksson U, Wolburg $H$, et al. Lack of pericytes leads to endothelial hyperplasia and abnormal vascular morphogenesis. J Cell Biol. 2001;153(3):543-53.

22. Park JE, Keller GA, Ferrara N. The vascular endothelial growth factor (VEGF) isoforms: differential deposition into the subepithelial extracellular matrix and bioactivity of extracellular matrix-bound VEGF. Mol Biol Cell. 1993;4(12):1317-26.

23. Paques $M$, Massin $P$, Gaudric A. Growth factors and diabetic retinopathy. Diabetes Metab. 1997;23(2):125-30.

24. Nagy JA, Benjamin L, Zeng H, Dvoral AM, Dvorak HF. Vascular permeability, vascular hyperpermeability and angiogenesis. Angiogenesis. 2008;11(2):109-19.
25. Grant DS, Kleinman HK, Goldberg ID, Bhargava MM, Nickoloff BJ, Kinsella JL, et al. Scatter factor induces blood vessel formation in vivo. Proc Natl Acad Sci U S A. 1993;90(5):1937-41.

26. Capp C, Zennig N, Wajner S, Maia AL. Papel do fator de crescimento endotelial vascular nos carcinomas de tireóide. Rev HCPA. 2009;29(1):51-9.

27. Ferrara N, Davis-Smyth T. The biology of vascular endothelial growth factor. Endocr Rev. 1997;18(1):4-25.

28. Vincenti V, Cassano C, Rocchi M, Persico G. Assignment of the vascular endothelial growth factor gene to human chromosome 6p21.3. Circulation. 1996;93(8):1493-5.

29. Tischer E, Mitchell R, Hartman T, Silva M, Gospodarowicz D, Fiddes JC, et al. The human gene for vascular endothelial growth factor. Multiple protein forms are encoded through alternative exon splicing. J Biol Chem. 1991;266(18):11947-54.

30. Woolard J, Wang WY, Bevan HS, et al. VEGF165b, an inhibitory vascular endothelial growth factor splice variant: mechanism of action, in vivo effect on angiogenesis and endogenous protein expression. Cancer Res. 2004;64(21):7822-35.

31. Quinn TP, Peters KG, De Vries C, Ferrara N, Williams LT. Fetal liver kinase 1 is a receptor for vascular endothelial growth factor and is selectively expressed in vascular endothelium. Proc Natl Acad Sci U S A. 1993;90(16):7533-7.

32. Pocock TM, Williams B, Curry FE, Bates DO. VEGF and ATP act by different mechanisms to increase microvascular permeability and endothelial $[\mathrm{Ca}(2+)](\mathrm{i})$. Am J Physiol Heart Circ Physiol. 2000;279(4):H1625-34.

33. Dawson NS, Zawieja DC, Wu MH, Granger HJ. Signaling pathways mediating VEGF165-induced calcium transients and membrane depolarization in human endothelial cells. Faseb J. 2006;20(7):991-3.

34. Gerber HP, Hillan KJ, Ryan AM, Kowalski J, Keller GA, Rangell L, et al. VEGF is required for growth and survival in neonatal mice. Development. 1999;126(6):1149-59.

35. Park JE, Chen HH, Winer J, Houck KA, Ferrara N. Placenta growth factor. Potentiation of vascular endothelial growth factor bioactivity, in vitro and in vivo, and high affinity binding to Flt-1 but not to Flk-1/KDR. J Biol Chem. 1994;269(41):25646-54.

36. Waltenberger J, Claesson-Welsh L, Siegbahn A, Shibuya M, Heldin $\mathrm{CH}$. Different signal transduction properties of KDR and Flt1, two receptors for vascular endothelial growth factor. J Biol Chem. 1994;269(43):26988-95.

37. Funatsu H, Yamashita H, Noma H, Mimura T, Yamashita T, Hori S. Increased levels of vascular endothelial growth factor and interleukin-6 in the aqueous humor of diabetics with macular edema. Am J Ophthalmol. 2002;133(1):70-7.

38. Funatsu $H$, Yamashita H, Shimizu E, Kojima R, Hori S. Relationship between vascular endothelial growth factor and interleukin- 6 in diabetic retinopathy. Retina. 2001;21(5):469-77.

39. Noma H, Funatsu H, Yamashita H, Kitano S, Mishima HK, Hori S. Regulation of angiogenesis in diabetic retinopathy: possible balance between vascular endothelial growth factor and endostatin. Arch Ophthalmol. 2002;120(8):1075-80.

40. Patel JI, Tombran-Tink J, Hykin PG, Gregor ZJ, Cree IA. Vitreous and aqueous concentrations of proangiogenic, antiangiogenic factors and other cytokines in diabetic retinopathy patients with macular edema: Implications for structural differences in macular profiles. Exp Eye Res. 2006;82(5):798-806.

41. Funatsu $H$, Yamashita $H$, Noma H, Shimizu E, Mimura T, Hori S. Prediction of macular edema exacerbation after phacoemulsification in patients with nonproliferative diabetic retinopathy. J Cataract Refract Surg. 2002;28(8):1355.

42. Chiarelli F, Santilli F, Mohn A. Role of growth factors in the development of diabetic complications. Horm Res. 2000;53(2):53-67.

43. Errera Fl, Canani LH, Silva ME, Yeh E, Takahashi W, Santos KG, et al. Functional vascular endothelial growth factor $-634 \mathrm{G}>\mathrm{C}$ SNP is 
associated with proliferative diabetic retinopathy: a case-control study in a Brazilian population of European ancestry. Diabetes Care 2007;30(2):275-9.

44. Kim HW, Ko GJ, Kang YS, Lee MH, Song HK, Kim HK, Cha DR. Role of the VEGF $936 \mathrm{C} / \mathrm{T}$ polymorphism in diabetic microvascular complications in type 2 diabetic patients. Nephrology (Carlton). 2009;14(7):681-8.

45. Kangas-Kontio T, Vavuli S, Kakko SJ, Penna J, Savolainen ER, Savolainen $\mathrm{MJ}$, et al. Polymorphism of the manganese superoxide dismutase gene but not of vascular endothelial growth factor gene is a risk factor for diabetic retinopathy. $\mathrm{Br} \mathrm{J}$ Ophthalmol. 2009;93(10):1401-6.

46. Nakanishi K, Watanabe C. Single nucleotide polymorphisms of vascular endothelial growth factor gene intron 2 are markers for early progression of diabetic retinopathy in Japanese with type 1 diabetes. Clin Chim Acta. 2009;402(1-2):171-5.

47. Nakamura S, Iwasaki N, Funatsu H, Kitano S, Iwamoto Y. Impact of variants in the VEGF gene on progression of proliferative diabetic retinopathy. Graefes Arch Clin Exp Ophthalmol. 2009;247(1):21-6.

48. Churchill AJ, Carter JG, Ramsden C, Turner SJ, Yeung A, Brenchley $P E$, et al. VEGF polymorphisms are associated with severity of diabetic retinopathy. Invest Ophthalmol Vis Sci. 2008;49(8):3611-6.

49. Petrovic MG, Korosec P, Kosnik M, Osredkar J, Hawlina M, Peterlin B, et al. Local and genetic determinants of vascular endothelial growth factor expression in advanced proliferative diabetic retinopathy. Mol Vis. 2008;14:1382-7.

50. Uthra S, Raman R, Mukesh BN, Rajkumar SA, Padmaja KR, Paul $P G$, et al. Association of VEGF gene polymorphisms with diabetic retinopathy in a south Indian cohort. Ophthalmic Genet. $2008 ; 29(1): 11-5$.
51. Szaflik JP, WysockiT, Kowalski M, Majsterek I, Borucka Al, Blasiak J, et al. An association between vascular endothelial growth factor gene promoter polymorphisms and diabetic retinopathy. Graefes Arch Clin Exp Ophthalmol. 2008;246(1):39-43.

52. Awata T, Kurihara S, Takata N, Neda T, lizuka H, Ohkubo T, et al. Functional VEGF C-634G polymorphism is associated with development of diabetic macular edema and correlated with macular retinal thickness in type 2 diabetes. Biochem Biophys Res Commun. 2005;333(3):679-85.

53. Ray D, Mishra M, Ralph S, Read I, Davies R, Brenchley P. Association of the VEGF gene with proliferative diabetic retinopathy but not proteinuria in diabetes. Diabetes. 2004;53(3):861-4.

54. Awata T, Inoue K, Kurihara S, Ohkubo T, Watanabe M, Inukai K, et al. A common polymorphism in the $5^{\prime}$-untranslated region of the VEGF gene is associated with diabetic retinopathy in type 2 diabetes. Diabetes. 2002;51(5):1635-9.

55. National Center for Biotechnology Information. Disponível em: http://www.ncbi.nlm.nih.gov/projects/SNP/snp_ref. cgi?rs=2010963. Acesso em: Abr 202010

56. Muniz JJ, Izidoro-Toledo TC, Metzger IF, Sandrim VC, Tanus-Santos JE. Interethnic differences in the distribution of clinically relevant vascular endothelial growth factor genetic polymorphisms. DNA Cell Biol. 2009;28(11):567-72.

57. Rasier R, Artunay O, Yuzbasioglu E, Sengul A, Bahcecioglu H. The effect of intravitreal bevacizumab (avastin) administration on systemic hypertension. Eye (Lond). 2009;23(8):1714-8.

58. Simo R, Hernandez C. Intravitreous anti-VEGF for diabetic retinopathy: hopes and fears for a new therapeutic strategy. Diabetologia. 2008;51(9):1574-80.

59. Mohamed Q, Gillies MC, Wong TY. Management of diabetic retinopathy: a systematic review. JAMA. 2007;298(8):902-16. 